\title{
Hydrothermal Growth of Zinc Oxide Nanorods and Glucose-Sensor Application
}

\author{
Shinji Nozaki ${ }^{1}$, Sachindra N. Sarangi ${ }^{1,2}$, Kazuo Uchida $^{1,2}$, Surendra N. Sahu ${ }^{3}$ \\ ${ }^{1}$ Graduate School of Informatics and Engineering, The University of Electro-Communications, Tokyo, Japan; ${ }^{2}$ Institute of Physics, \\ Sachivalaya Marg, Bhubaneswar, India; ${ }^{3}$ Center for Nanoscience and Technology, National Institute of Science \& Technology, Palur \\ Hills, Berhampur, India. \\ Email: nozaki@ee.uec.ac.jp
}

Received October $1^{\text {st }}, 2013$; revised November $7^{\text {th }}, 2013$; accepted November $14^{\text {th }}, 2013$

Copyright (C) 2013 Shinji Nozaki et al. This is an open access article distributed under the Creative Commons Attribution License, which permits unrestricted use, distribution, and reproduction in any medium, provided the original work is properly cited.

\begin{abstract}
High-quality zinc oxide nanorods were grown on various substrates using zinc nitrate $\left(\mathrm{Zn}\left(\mathrm{NO}_{3}\right)_{2}\right)$ and hexamethylenetetramine $\left(\left(\mathrm{CH}_{2}\right)_{6} \mathrm{~N}_{4}\right)$. The substrates greatly affect the hydrothermal growth of $\mathrm{ZnO}$ nanorods. Making the best use the substrate effect, we engineered substrates to make a single nanorod in each hole of $100 \mathrm{~nm} \times 100 \mathrm{~nm}$ in the array of the holes on the photoresist-patterned substrate. It is also interesting to note that high-quality $\mathrm{ZnO}$ nanorods grown on $\mathrm{GaN}$ substrates by the hydrothermal growth technique have demonstrated the potential application as a glucose sensor without oxidase for the first time. The photoluminescence in the UV wavelength range was quenched by immobilizing glucose on the $\mathrm{ZnO}$ surface. The peak intensity decreased increased with the increased glucose concentrations. A good linearity and high sensitivity were obtained for the glucose concentrations of $0.5-30 \mathrm{mM}$ in the calibration curve. The calibration curve was not influenced by the presence of bovin serum albumin (BSA), ascorbic acid (AA) and uric acid (UA), which are also included in human blood and could cause interference in estimating glucose concentrations in human blood. The PL quenching was attributed to the $\mathrm{H}_{2} \mathrm{O}_{2}$ molecules, which were produced by the photo-oxidation of glucose during exposure to UV light. The PL-quenching glucose sensor made of $\mathrm{ZnO}$ nanorods has been evaluated for the first time by estimating the glucose concentrations in the human serum samples which include those of diabetes, and a good correlation was obtained between the concentrations by the PL quenching and the clinical data provided by a local hospital.
\end{abstract}

Keywords: ZnO; Hydrothermal Growth; Glucose Sensor; Photoluminescence Quenching

\section{Introduction}

Zinc oxide $\mathrm{ZnO}$ nanorods have attracted the increasing attention because of their wide variety of electronic and photonic device applications as a wide band gap semiconductor. One of interesting applications is a UV LED made of $n-\mathrm{ZnO}$ and $p-\mathrm{ZnO}$. Since it is difficult to form $p$ - $\mathrm{ZnO}$, it may be replaced by $p$ - $\mathrm{NiO}$ as a $p$-type material in the $p n$ diode. $\mathrm{NiO}$ is known to be a p-type wide band gap semiconductor [1], and an ultraviolet-detector was made of a $p-\mathrm{NiO} / n-\mathrm{ZnO}$ [2]. However, it is expected to improve the diode characteristic by using $\mathrm{ZnO}$ nanorods, which usually have crystallinity better than $\mathrm{ZnO}$ thin films because of the 1D growth. Utilizing the effect of substrate, $\mathrm{ZnO}$ nanorods can be formed selectively on the engineered substrate by the hydrothermal growth technique.
In addition, $\mathrm{ZnO}$ nanorods have great application potential in the field of biosensors due to their excellent biocompatibility, optical property, non-toxicity, chemical and electrochemical stability, high electron communication features and large specific surface area. The currently available sensors are based on electrochemical principles where the enzyme glucose oxidase serves as a molecular recognition element. Glucose can be converted in hydrogen peroxide and gluconic acid under oxygen consumption catalyzed by glucose oxidase. Hence, enzymetic electrochemical/amperometric glucose sensors based on glucose oxidase $\left(\mathrm{GO}_{\mathrm{x}}\right)$, have played a leading role in blood sugar testing. The $\mathrm{GO}_{\mathrm{x}}$, is a widely used analytical enzyme for glucose detection. In spite of the many impressive advances in the design and use of enzymatic glucose biosensors, yet, the promise of reliable 
and accurate glucose sensing has not been fulfilled. There are still major challenges in achieving a stable, clinically accurate glucose monitoring. Many attempts have been made to fabricate glucose biosensors using $\mathrm{ZnO}$ [3-7] nanowires, nanorods and nanoparticles etc. to estimate glucose concentrations in human blood. Most of them are based on enzymatic and electrochemical techniques. However, these sensors are limited to their calibration range, biosensor response time, lifetime, stability etc. Luminescence quenching is a phenomenon that when the molecules are attached to the surface of the host material, the luminescence intensity of the host material decreases with the increased concentration of the immobilized molecules [6,7]. The luminescence quenching is usually understood in terms of the electron transfer reaction from the photo-excited particles to electron absorbing acceptors. It suggests that electron or hole acceptors adsorbed at the surface of nanostructures can change their luminescence properties and quench the exciton emission by fast electron transfer. We report non-enzymatic glucose sensing by PL emission quenching technique in $\mathrm{ZnO}$ nanorods and discuss their potential application as a glucose biosensor using $\beta$-D-glucose.

\section{Experimental}

Aligned $\mathrm{ZnO}$ nanorods were grown on GaN substrates with an area of about $1.0 \mathrm{~cm}^{2}$ by the hydrothermal growth technique using a mixture of $10 \mathrm{mM}, \mathrm{Zn}$ $\left(\mathrm{NO}_{3}\right)_{2} \cdot 6 \mathrm{H}_{2} \mathrm{O}$ and $\left(\mathrm{CH}_{2}\right)_{6} \mathrm{~N}_{4}$. The details of the growth have been described elsewhere [8]. Aqueous solutions of the $\beta$-D glucose $(\beta$ form of dextrose, mol. wt. $180.16 \mathrm{~g}$, $99 \%$ pure) purchased from Merck, were prepared in the concentration range of $0.25 \mathrm{mM}$ to $40 \mathrm{mM}$, and an amount of $20 \mu \mathrm{L}$ taken from each solution was drop casted on the $\mathrm{ZnO}$ nanorods to immobilize the glucose on the $\mathrm{ZnO}$ nanorod surface. The morphology and structure of $\mathrm{ZnO}$ Nanorods were studied by scanning electron microscopy (SEM) and X-ray diffraction (XRD) measurement. The photoluminescence (PL) spectra of the $\mathrm{ZnO}$ nanorods without glucose and with various concentrations of glucose were collected at $300 \mathrm{~K}$ using an Oriel PL system (SPEC-TRACQ2) with a single monochrometer and PMT detector. A wavelength of $250 \mathrm{~nm}$ was selected for excitation with a single monochromator from the spectrum of a $\mathrm{Hg}$-Xe lamp.

\section{Results and Discussion}

Figure 1(a) shows high density, well aligned $\mathrm{ZnO}$ nanorods vertically grown on GaN substrate. The $\mathrm{ZnO}$ nanorods deposited on GaN were oriented along the (002) direction of the hexagonal lattice, as shown in Figure 1(b).

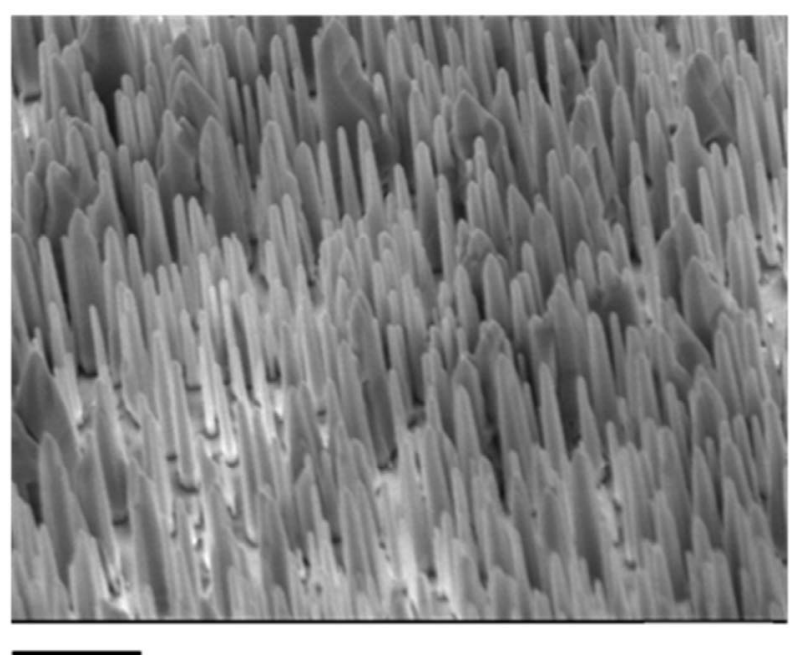

$1 \mu \mathrm{m}$

(a)

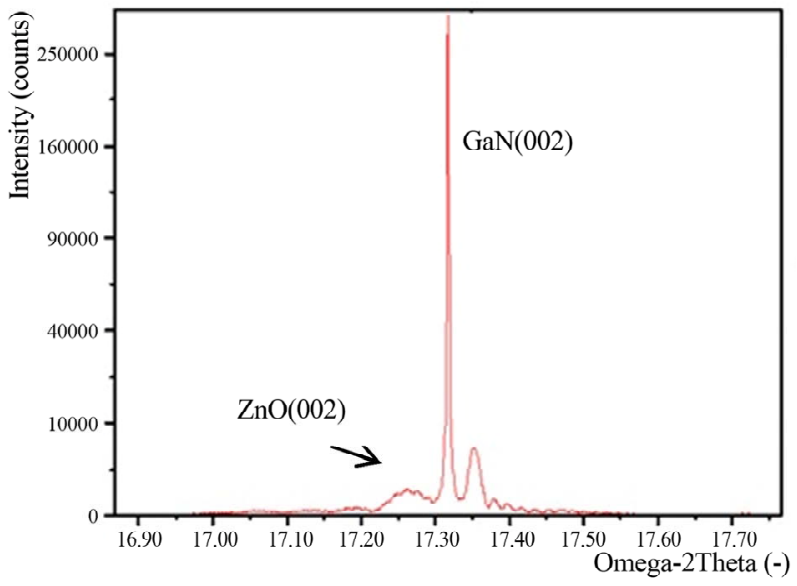

(b)

Figure 1. (a) SEM image and (b) XRD pattern of ZnO nanorods grown on $\mathrm{GaN}$.

The photoresist on GaN was patterned to make an array of holes on a GaN-on-sapphire substrate by e-beam lithography, and then the hydrothermal growth of $\mathrm{ZnO}$ nanorods was carried out. More than one nanorod was grown when the hole size is $700 \mathrm{~nm} \times 700 \mathrm{~nm}$, while only one single nanorod was grown in a hole when the hole size decreased to $200 \mathrm{~nm} \times 200 \mathrm{~nm}$. However, they were not grown in all holes after $1 \mathrm{hr}$ growth, as shown in Figure 2(a). A single nanorod was successfully grown in every hole of the sample by decreasing the concentration to $5 \mathrm{mM}$ and increasing the growth time to $16 \mathrm{hrs}$, as shown in Figure 2(b). It is also noted that a lower concentration increases the aspect ratio.

Figure 3(a) shows the PL spectra of $\mathrm{ZnO}$ nanorods on $\mathrm{GaN}$ treated with the glucose of various concentrations in the UV wavelength range. The intensity is normalized with respect to the peak intensity of the as-grown $\mathrm{ZnO}$ nanorods. The PL spectrum of the as-grown $\mathrm{ZnO}$ nano- 


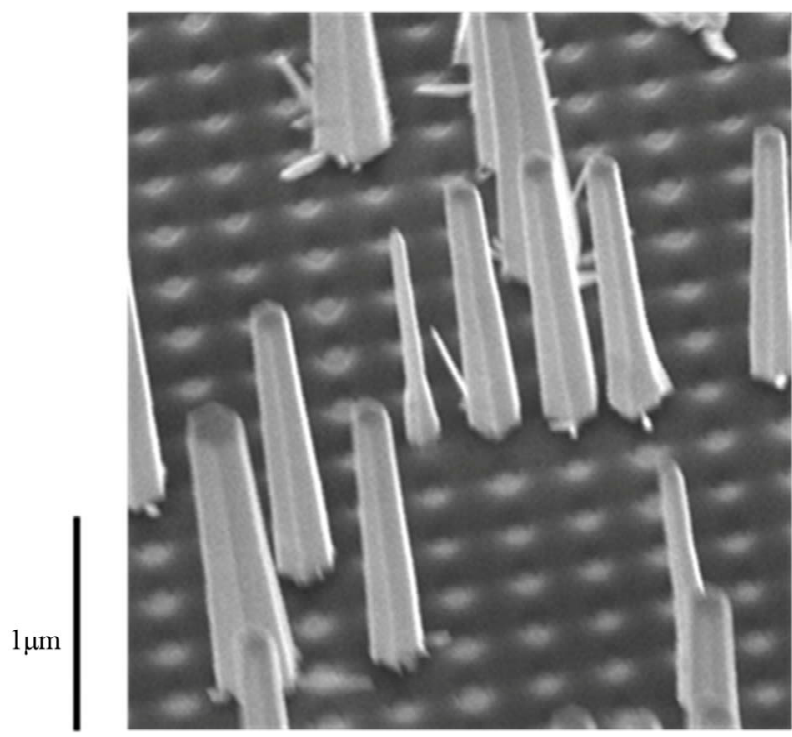

(a)

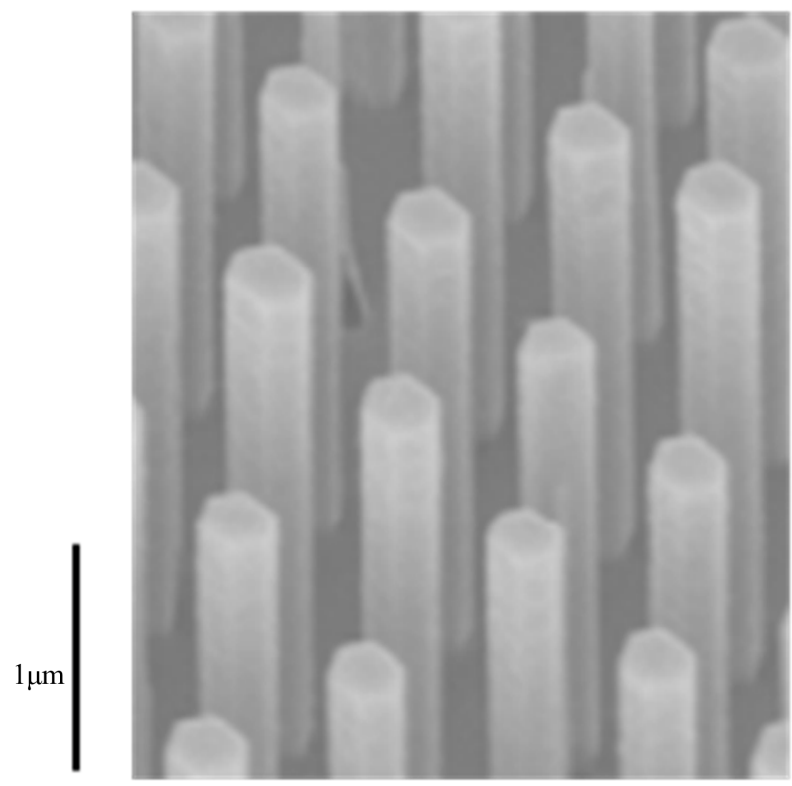

(b)

Figure 2. Selective epitaxial growth of $\mathrm{ZnO}$ nanorods on the photoresist-patterned GaN. (a)1 hr, 10 mM; 16 hrs, 5 mM.

rods consists of two peaks, one in the UV range and the other in the visible wavelength region [8]. The narrower $\mathrm{PL}$ peak at $377 \mathrm{~nm}$ is associated with the near bandgap emission, while the broader peak at $580 \mathrm{~nm}$ is associated with point defects. Since the peak position and intensity vary in the visible wavelength region among the $\mathrm{ZnO}$ nanorod samples, but less in the UV region, we studied the quenching of PL in the UV region by the glucose attachment.

As seen in the figure, the PL peak intensity of the $\mathrm{ZnO}$ nanorods decreases with the increased glucose concentration without much change in the peak wavelength, and
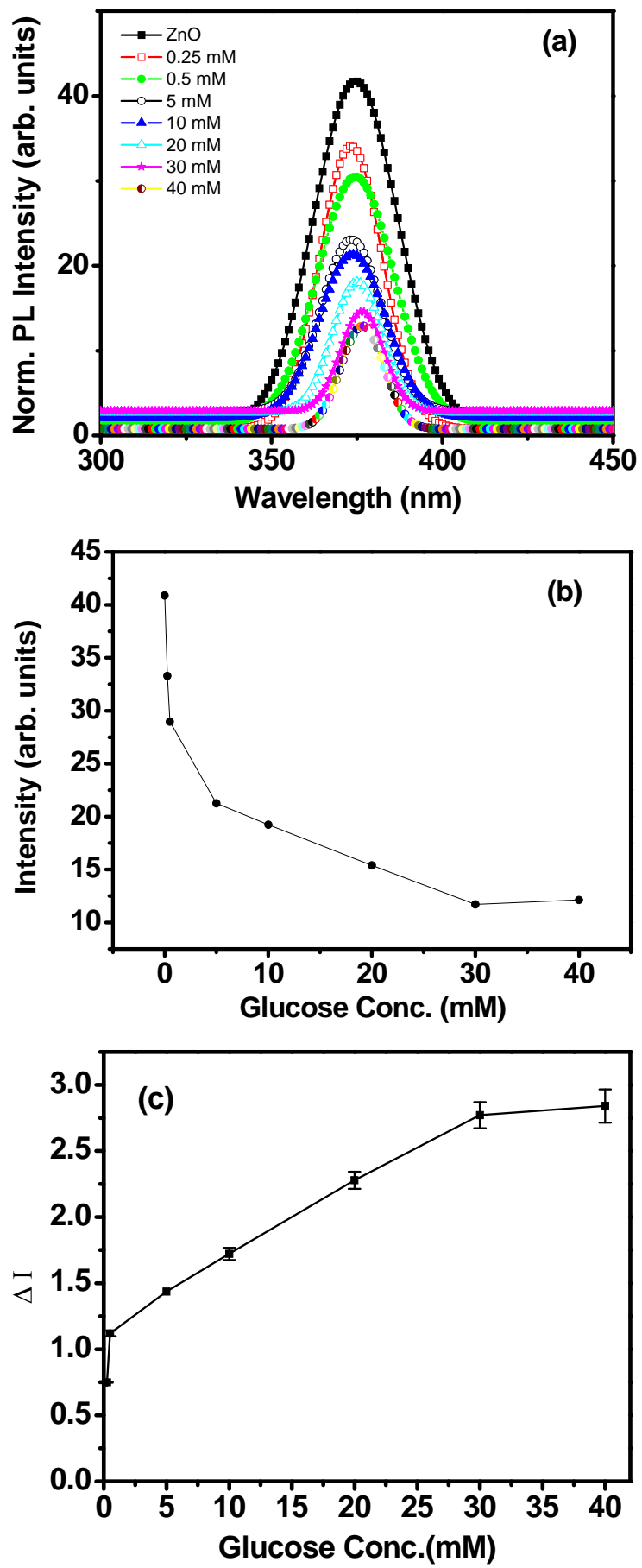

Figure 3. (a) Photoluminescence spectra of $\mathrm{ZnO}$ nanorods treated with glucose of various concentrations; (b) shows the PL peak intensity of $\mathrm{ZnO}$ nanorods as a function of the glucose concentration, and (c) is the calibration plot obtained from (b).

does not further decrease for concentrations higher than $30 \mathrm{mM}$. A slight red shift in the peak is also observed with the increased concentration for concentrations of 30 $\mathrm{mM}$ or higher. Note that the PL rapidly quenches during the initial stage, and this was followed by a gradual de- 
crease with the increased glucose concentration.

Figure 3(b) shows the PL peak intensity for various glucose concentrations, and Figure 3(c) is obtained from Figure 3(b) as a calibration plot. A good linearity is obtained in the glucose concentration range of $0.5 \mathrm{mM}$ to $30 \mathrm{mM}$, corresponding to $9-540 \mathrm{mg} / \mathrm{dL}$, as shown in the calibration plot. The sensor sensitivity, which is defined as the slope of the line, is found to be $1.4 \% / \mathrm{mM}$ with a correlation coefficient of 0.99 . The sensing range of the sensor is well suitable for a glucose sensor of human blood.

Several possible mechanisms for the PL quenching have been proposed. The surface reaction with a quencher may introduce the nonradiative surface defects. The charge transfer from a radiative material to a quencher was also proposed to be the main mechanism of PL quenching in many studies. Kim et al. recently observed the PL quenching of enzyme-conjugated $\mathrm{ZnO}$ nanocrystals treated with glucose and proposed $\mathrm{H}_{2} \mathrm{O}_{2}$ as a quencher [9]. The PL quenching occurred when the radiative transition of the excited electrons were suppressed by a quencher. The energies of the valence band and conduction band edges increased by the quantum confinement facilitated the electron transfer from the $\mathrm{ZnO}$ to $\mathrm{H}_{2} \mathrm{O}_{2}$. Since the amount of the PL quenching was proportional to the $\mathrm{H}_{2} \mathrm{O}_{2}$ concentration, which was also proportional to the glucose concentration, the PL quenching could be used as a measure of the glucose concentration. The PL quenching observed in Figure 3(a) is similar to that of the enzyme-conjugated $\mathrm{ZnO}$ nanoparticles. However, as mentioned in the Introduction, in the enzymatic sensor, the glucose oxidase is needed as a catalyst to oxidize glucose and produce gluconic acid and $\mathrm{H}_{2} \mathrm{O}_{2}$. In our case, the glucose oxidase is absent, and no catalysis is present to oxidize the glucose. $\mathrm{ZnO}$ nanorods themselves act as a catalyst to oxidize the glucose.

\section{Conclusion}

One single nanorod was successfully grown by the hydrothermal growth technique in every hole of the photoresist-patterned GaN when the hole size was 200 $\mathrm{nm} \times 200 \mathrm{~nm}$ or less and the solution concentration was reduced to $5 \mathrm{mM}$. The $\mathrm{ZnO}$ nanorods grown on $\mathrm{GaN}$ by the hydrothermal growth technique show strong near band-edge PL, which is quenched by immobilizing glucose on the surface of $\mathrm{ZnO}$ nanorods. The amount of the decrease in the PL peak intensity increases with the increased glucose concentrations, and the calibration curve shows a good linearity with a sensor sensitivity of $1.4 \% / \mathrm{mM}$ over the wide range of $0.5-30 \mathrm{mM}$, corresponding to $9-540 \mathrm{mg} / \mathrm{dL}$. A high sensitivity and good lineality over the wide range make the use of $\mathrm{ZnO}$ nanorods highly favorable for a glucose-biosensor application. Furhtermore, the $\mathrm{ZnO}$ nanorod glucose sensor does not require an oxidase to oxidize glucose, because the $\mathrm{ZnO}$ nanorods themselves act as photocatalyses.

\section{Acknowledgements}

This project was in part supported by the Indo-JSPS project (DST-JSPS Project No. DST/JAP/P-68/09). S. N. $\mathrm{S}$ greatly acknowledges the JSPS Postdoctoral Research Fellowship for Foreign Researchers.

\section{REFERENCES}

[1] D. Alders, F. C. Voogt, T. Hibma and G. A. Sawatzky, "Nonlocal Screening Effects in 2p X-ray Photoemission Spectroscopy of NiO (100)," Physical Review B, Vol. 54, No. 11, 1996, pp. 7716-7719.

http://dx.doi.org/10.1103/PhysRevB.54.7716

[2] H. Ohta, M. Hirano, K. Nakahara, H. Maruta, Tanabe, M. Kamiya, T. Kamiya and H. Hosono, "Fabrication and Photoresponse of a pn-Heterojunction Diode Composed of Transparent Oxide Semiconductors, p-NiO and n-ZnO," Applied Physics Letters, Vol. 83, No. 5, 2003, p. 1029. http://dx.doi.org/10.1063/1.1598624

[3] A. Wei, X. W. Sun, J. X. Wang, Y. lie, X. P. Cai, C. M. Li, Z. L. Dong and W. Huang, "Enzymatic Glucose Biosensor Based on $\mathrm{ZnO}$ Nanorod Array Grown by Hydrothermal Decomposition," Applied Physics Letters, Vol. 89, No. 12, 2006, pp. 123902-123904. http://dx.doi.org/10.1063/1.2356307

[4] H. Liu, X. Xian, S. Wang, Y. Li, Y. Song and D. Zhu, "Ultra-Sensitivity Glucose Sensor Based on Field Emitters" Nanoscale Research Letters, Vol. 4, 2009, pp. 11411145. http://dx.doi.org/10.1007/s11671-009-9372-0

[5] M. H. Asif, S. M. U. Ali, O. Nur, M. Willander, C. Brannmark, P. Stralfors, U. H. Englund, F. Elinder and B. Danielsson, "Functionalised ZnO-Nanorod-Based Selective Electrochemical Sensor for Intracellular Glucose," Biosensors and Bioelectronics, Vol. 25, No. 10, 2010, pp. 2205-2211. http://dx.doi.org/10.1016/j.bios.2010.02.025

[6] X. Q. Yan, Z. B. Shang, Z. Zhang, Y. Wang and W. J. Jin, "Fluorescence Sensing of Nitric Oxide in Aqueous Solution by Triethanolamine-modified CdSe Quantum Dots," Luminescence, Vol. 24, No. 4, 2009, pp. 255-259. http://dx.doi.org/10.1002/bio.1109

[7] A. Merkoç, "Nanoparticles-Based Strategies for DNA, Protein and Cell Sensors" Biosensors and Bioelectronics, Vol. 26, No. 4, 2010, pp. 1164-1177. http://dx.doi.org/10.1016/j.bios.2010.07.028

[8] J. Nayak, S. N. Sahu, J. Kasuya and S. Nozaki, "Effect of Substrate on the Structure and Optical Properties of $\mathrm{ZnO}$ Nanorods," Journal of Physics D: Applied Physics, Vol. 41, No. 11, 2008, pp. 115303-115308. http://dx.doi.org/10.1088/0022-3727/41/11/115303

[9] K. Kim, T. G. Kim and Y. Sung, "Enzyme-Conjugated ZnO Nanocrystals for Collisional Quenching-Based Glucose Sensing," CrystEngComm, Vol. 14, No. 8, 2012, pp. 2859-2865. http://dx.doi.org/10.1039/c2ce06410c 